\title{
MATHEMATICAL CHALLENGES IN THE THEORY OF CHEMOTAXIS
}

\author{
Piotr BiLer
}

The XI Annual Lecture dedicated to the memory of Professor Andrzej Lasota

\begin{abstract}
We consider the simplest parabolic-elliptic model of chemotaxis in the whole space and in several space dimensions. Criteria either for the existence of radial global-in-time solutions or their blowup in terms of suitable Morrey spaces norms are discussed.

This is an extended version of the lecture presented at the University of Silesia on January 12, 2018, commemorating Professor Andrzej Lasota — great scholar, master of fine mathematics and applications to real world.
\end{abstract}

\section{Introduction}

We discuss in this paper some intriguing properties of solutions of the following Cauchy problem in space dimensions $d \geq 2$ :

$$
\begin{aligned}
u_{t}-\Delta u+\nabla \cdot(u \nabla v) & =0, \quad x \in \mathbb{R}^{d}, t>0, \\
\Delta v+u & =0, \quad x \in \mathbb{R}^{d}, t>0, \\
u(x, 0) & =u_{0}(x), \quad x \in \mathbb{R}^{d} .
\end{aligned}
$$

Received: 22.02.2018. Accepted: 21.03.2018. Published online: 14.05.2018.

(2010) Mathematics Subject Classification: 35Q92, 35B44, 35K55.

Key words and phrases: chemotaxis, blowup of solutions, global existence of solutions.

The author was partially supported by the NCN grant 2016/23/B/ST1/00434. 
There are many motivations to study this seemingly simple diffusion-transport model. One of them comes from Mathematical Biology, where equations (1.1)(1.2) appear as a simplified Keller-Segel(-Patlak) (the, so called, minimal KS) system modelling chemotaxis, see e.g., the seminal paper [50, overviews [4, [44, 45, 43] and [8, 33, 53, 55, 54]. The unknown variables $u=u(x, t)$ and $v=v(x, t)$ denote the density of the population of microorganisms (e.g., swimming bacteria or slime mold), and the density of the chemical secreted by themselves that attracts them and makes them to aggregate, respectively.

Another and even earlier important interpretation of system (1.1)-(1.2) stems from astrophysics, where the unknown function $u=u(x, t)$ is the density of gravitationally interacting massive particles in a cloud (of molecules, stars, nebulae, etc.), and $v=v(x, t)$ is the Newtonian potential ("mean field") of the mass distribution $u$, see [34, 35, 5, 7, 6, 18. Note that similar mean field models, with + sign in equation 1.2 replaced by - sign have been used for more than one century to model migration of electrically charged particles in electrolytes, plasma and semiconductors, see e.g., [37] and [16] for further references.

The initial data $(1.3)$ are nonnegative integrable functions $u_{0} \in L^{1}\left(\mathbb{R}^{d}\right)$. The total mass $M=\int u_{0}(x) \mathrm{d} x=\int u(x, t) \mathrm{d} x \in[0, \infty)$ is conserved during the evolution. Further, we will also consider solutions with infinite mass like the famous Chandrasekhar steady state singular solution in [34] related to black holes

$$
u_{C}(x)=\frac{2(d-2)}{|x|^{2}} .
$$

In fact, a more realistic description of chemotaxis phenomena for biologists needs more complicated models involving nonlinear diffusion, growth and reaction terms and nonlinear sensitivity functions like (here $\varphi \equiv v$ )

$$
\begin{aligned}
u_{t} & =\nabla \cdot(D(u) \nabla u-u \nabla \chi(\varphi))+g(u, \varphi), \\
\varepsilon \varphi_{t} & =\Delta \varphi+u+h(u, \varphi),
\end{aligned}
$$

where, for instance,

$$
\begin{gathered}
\varepsilon \geq 0, \quad D(u)=d u^{\nu}, \quad D(u) \asymp u\left(1+u^{\lambda}\right), \\
\chi(\varphi)=c \log \varphi, \quad \chi(\varphi)=c \varphi^{\kappa}, \quad \chi(\varphi)=\frac{\varphi}{c+\varphi}, \\
g(u, \varphi)=\mu u(1-u) ; \quad h(u, \varphi)=-\gamma \varphi, \ldots
\end{gathered}
$$


Another class of biologically relevant models appear with the following choice for the first equation

$$
u_{t}=\nabla \cdot(D(u) \nabla u-u h(u) \nabla \varphi)
$$

where $D(s)=h(s)-s h^{\prime}(s)$. The literature on these subjects is abundant and fast growing.

We will concentrate on the simplified system (1.1) -1.2 which deserves a deep analysis by mathematicians since this features many interesting behaviors of solutions.

This is a review paper, a potpourri of some old results, some new, with no proofs - except for a sketch of one being an application of a classical idea of H. Fujita 38 to radial solutions of chemotaxis systems.

The system 1.1 - 1.2 has a variational structure, so that the quantity (of a clear physical origin of "entropy" or "free energy")

$$
W(t)=\int u \log u \mathrm{~d} x-\frac{1}{2} \int u \varphi \mathrm{d} x
$$

is a Lyapunov functional

$$
\frac{\mathrm{d}}{\mathrm{d} t} W=-\int u|\nabla \log u-\nabla \varphi|^{2} \mathrm{~d} x \leq 0 .
$$

However, unlike the authors of [3, 32, 30], we had not used that subtle property in the proofs of our results presented here.

Finally, it should be noted that similar phenomena take place and can be proved for nonnegative solutions (not necessarily radial) of the nonlinear heat equation

$$
u_{t}=\Delta u+u^{p}
$$

A general reference is the monograph [61, and recent results are in [63, 10, 11].

\section{The $8 \pi$-problem in the two-dimensional case}

Let us now describe previous results which motivated us to start this study and we limit ourselves to those publications, which are directly related to that topic.

We begin with the classical case of $d=2$ where the value $M=8 \pi$ of mass plays a crucial role. Namely, if $u_{0}$ is a nonnegative measure of mass $M<8 \pi$ 
(the subcritical case), then there exists a unique solution which is globalin-time and bounded (see, e.g., [3, 32, 28]) and its asymptotics is essentially selfsimilar in space-time. These results have been known previously for radially symmetric initial data, see [21, 22, 9, 24, 25] for recent presentations.

On the other hand, if $M>8 \pi$ (the supercritical case), then this solution cannot be continued to a global-in-time regular one, and a finite time blowup occurs

$$
\limsup _{t \nearrow T, x \in \mathbb{R}^{d}} u(x, t)=\infty \quad \text { for some } \quad 0<T<\infty .
$$

The first proof of blowup was in [47], then [27, 18, 6, 9, 56, 52] appeared, and constructions of blowing up radial solutions have been presented in [41, 42]. The radial blowup is accompanied by the concentration of mass equal to $8 \pi$ at the origin.

The book 64] is devoted to a fine description of solutions at the blowup time, in particular there is a quantization of mass property presented: the local singularities of blowing up solutions eventually grow to integer multiples of $8 \pi$.

These phenomena are closely related to the question of local solvability of the Cauchy problem for system (1.1) 1.2 under minimal regularity on the initial data $u_{0} \geq 0$ in 1.3 . Namely, if $u_{0}$ is a nonnegative measure then local in time solution exists if and only if all the atoms of $u_{0}$ have mass less than $8 \pi$, see [3] and [28] for much simpler argument.

The critical case $M=8 \pi$ is rich in fine asymptotic behavior results (see [31, 30]) even in the radially symmetric case. And the case of a ball [49, 21 is quite different from the case of the whole plane in [22]. The study of radially symmetric solutions of system (1.1) 1.2 can be reduced to a nonlinear equation which is no longer nonlocal

$$
M_{t}=4 s M_{s s}+\frac{1}{\pi} M M_{s}
$$

for the nondecreasing radial distribution function

$$
M(r, t)=\int_{\{|x|<r\}} u(x, t) \mathrm{d} x,
$$

after the change of variables $s=r^{2}$. Similarly, for $d \geq 3$ and $s=r^{d}$ equation

$$
M_{t}=d^{2} s^{2-2 / d} M_{s s}+\frac{d}{\sigma_{d}} M M_{s}
$$


appears with

$$
\sigma_{d}=\frac{2 \pi^{\frac{d}{2}}}{\Gamma\left(\frac{d}{2}\right)}
$$

denoting the area of the unit sphere $\mathbb{S}^{d-1}$ in $\mathbb{R}^{d}$. These equations feature nonuniform diffusion coefficients $s^{2-2 / d}$ and simple nonlinear terms (as in the Burgers equation corresponding to $d=1$ case of 2.3 with the uniform diffusion coefficient). Equation (2.1) has infinite number of steady states given by formula

$$
M_{b}(s)=8 \pi \frac{s}{s+b}, \quad b>0,
$$

having the property

$$
\int_{0}^{\infty}\left(8 \pi-M_{b}(s)\right) \mathrm{d} s=\infty \text { for } \quad b>0 .
$$

They are locally asymptotically stable which is shown by considering relative entropy functionals

$$
\begin{gathered}
\mathcal{W}_{b}(M)=\int\left(M \log \frac{M}{M_{b}}+(8 \pi-M) \log 8 \pi-M 8 \pi-M_{b}\right) \mathrm{d} s \\
\mathcal{W}_{b}\left(M_{0}\right)<\infty \Longrightarrow \lim _{t \rightarrow \infty}\left\|M(t)-M_{b}\right\|_{L^{1}(0, R)}=0 \quad \text { for each } \quad R>0,
\end{gathered}
$$

as in [22]. But the global dynamics picture is much more complicated; there are solutions which diffuse mass to infinity, so that $M(r, t) \rightarrow 0$ as $t \rightarrow \infty$, solutions with an infinite time blowup that concentrate at the origin: $M(r, t) \rightarrow 8 \pi$ for all $r>0$ as $t \rightarrow \infty$, and solutions that oscillate ("bounce") between two different steady states $M_{b}$, see [58].

The doubly parabolic case of Keller-Segel system, i.e., equation (1.1) supplemented with the linear parabolic diffusion equation for $\varphi$

$$
\varepsilon \varphi_{t}=\Delta \varphi+u
$$

instead of 1.2 , is even more difficult to study, especially when blowup questions are considered. A striking difference of its behavior is, e.g., result in [15]. Namely, selfsimilar solutions satisfying scaling property

$$
u(x, t)=t^{-1} U\left(x t^{-1 / 2}\right), \quad \varphi(x, t)=\Phi\left(x t^{-1 / 2}\right),
$$


exist for each $0 \leq M<M_{\varepsilon}$ with the optimal value of $M_{\varepsilon}=8 \pi$ whenever $0 \leq \varepsilon \leq \frac{1}{2}$, while $\lim _{\varepsilon \rightarrow \infty} M_{\varepsilon}=\infty$. These are solutions of the Cauchy problem (1.1)-(1.2) with $u_{0}=M \delta_{0}$, nonunique if $M>8 \pi$.

The role of consumption term $\gamma \varphi$ in the modified equation 2.5) for the chemoattractant $\varepsilon \varphi_{t}=\Delta \varphi-\gamma \varphi+u$ is discussed in [17] for two-dimensional doubly parabolic model (together with the dependence on diffusivity coefficient $\varepsilon>0)$ and in [14] in the parabolic-elliptic case $(\varepsilon=0)$. Namely, for each initial condition there is $\varepsilon_{0}=\varepsilon\left(u_{0}\right)$ such that for $\varepsilon \geq \varepsilon_{0}\left(\gamma_{0}=\gamma\left(u_{0}\right)\right.$ and for $\gamma \geq \gamma_{0}$, resp.) solution with $u_{0}$ as the initial datum is global in time.

\section{Parabolic-elliptic model in higher dimensions}

In view of results in the two-dimensional case mentioned above (when the parameter of total mass $M$ plays decisive role in the temporal behavior of solutions), for $d \geq 3$ we are looking for a critical quantity $\tilde{\ell}=\tilde{\ell}\left(u_{0}\right)$ which decides about the blowup. More precisely, do there exist constants $0<c(d) \leq$ $C(d)$ such that $\tilde{\ell}\left(u_{0}\right)<c(d)$ implies global-in-time existence of solution to 1.1 -1.2 while $\tilde{\ell}\left(u_{0}\right)>C(d)$ leads to a finite time blowup of solution? We will give an answer to that dichotomy question in Corollary 5.4 showing that $\tilde{\ell}$ is close to the radial concentration - and thus equivalent to the Morrey norm in the space $M^{d / 2}\left(\mathbb{R}^{d}\right)$. A generalization to the case of the dissipation defined by a fractional power of Laplacian $(-\Delta)^{\alpha / 2}$ with $\alpha \in(1,2)$ is in a forthcoming paper [20], showing that $\tilde{\ell}$ is close to the Morrey norm in the space $M^{d / \alpha}\left(\mathbb{R}^{d}\right)$. Here, the radial concentration of a locally integrable radial function $u \geq 0$ is defined by

$$
\|u\|=\sup _{R>0} R^{2-d} \int_{\{|y|<R\}} u(y) \mathrm{d} y .
$$

The homogeneous Morrey spaces of measures on $\mathbb{R}^{d}$ are defined by their norms

$$
|u|_{M^{p}} \equiv \sup _{R>0, x \in \mathbb{R}^{d}} R^{d(1 / p-1)} \int_{\{|y-x|<R\}}|u(y)| \mathrm{d} y<\infty .
$$

Clearly, $\|u\| \leq|u|_{M^{d / 2}}$ but in fact these quantities are equivalent for $d \geq 3$, see [26, Lemma 7.1] as well as [1, Lemma 3.1]. 


\section{Main new results}

Our main results obtained recently include:

- global-in-time existence of radially symmetric solutions with initial data in the critical Morrey space $M^{d / 2}\left(\mathbb{R}^{d}\right)$ whose initial conditions are uniformly below the singular solution $u_{C}$ in (1.4) in an averaged sense, Theorem 4.1 below, together with their convergence to 0 as $t \rightarrow \infty$;

- sufficient conditions on the radial initial data which lead to a finite time blowup of solutions, expressed in terms of quantities related to the Morrey space norm $M^{d / 2}\left(\mathbb{R}^{d}\right)$, Theorem 5.1; for instance, condition (5.7):

$$
\sup _{T>0} T \mathrm{e}^{T \Delta} u_{0}(0)>C(d)
$$

(here, $\mathrm{e}^{T \Delta}$ denotes the heat semigroup on $\mathbb{R}^{d}$ ) is sufficient for the blowup of solution with the initial condition $u_{0}$;

- a perturbation result on convergence of a solution $u(t)$ to $u_{C}$ as $t \rightarrow \infty$ studied via the hypercontractivity property of the semigroup linearized at $u_{C}$ in high dimensions $d \geq 15$ (in the radial case), and for $d \geq 17$ (with neither symmetry nor sign assumptions) in Section 6 .

The proof of the first result involves a pointwise argument, a powerful tool used in different contexts such as free boundary problems and fluid dynamics, cf. also [10] for the case of a nonlinear heat equation. A sufficient condition for the global-in-time existence is, in fact, an estimate of the Morrey space $M^{d / 2}\left(\mathbb{R}^{d}\right)$ norm of the initial condition (modulo a mild regularity assumption).

For the proof of the second result, we revisit a classical argument of $\mathrm{H}$. Fujita (applied to the nonlinear heat equation in [38]) and reminiscent of ideas in 33. This leads to a sufficient condition for blowup of radially symmetric solutions of system (1.1)-(1.2), with a significant improvement compared to [26] where local moments have been employed. Then, we derive as corollaries of condition (5.7) other criteria for blowup of solutions of (1.1)-(1.3).

Remark 5.3 deals with the initial trace of a nonnegative solution of the Keller-Segel system, and again the Morrey space $M^{d / 2}\left(\mathbb{R}^{d}\right)$ norm enters as a critical quantity which measures the minimal regularity of the initial data needed for the existence of a local-in-time solution of that system.

\section{Local- and global-in-time solutions}

It is well-known that problem (1.1)- 1.3 has a unique local-in-time mild solution $u \in \mathcal{C}\left([0, T) ; L^{p}\left(\mathbb{R}^{d}\right)\right)$ for every $u_{0} \in L^{p}\left(\mathbb{R}^{d}\right)$ with $p>d / 2$, see [5, 48, 51]. For solvability results in other functional spaces like weak Lebesgue (Marcinkiewicz), Morrey and Besov spaces, see also [13, 36, 48, 53] where 
the classical Fujita-Kato iterations procedure for construction of mild solutions is used. Mild solutions are those which are weakly continuous in $t$ : $u \in \mathcal{C}_{\mathrm{w}}\left([0, T) ; M^{d / 2}\left(\mathbb{R}^{d}\right) \cap M^{p}\left(\mathbb{R}^{d}\right)\right)$ and satisfy the Duhamel formula

$$
u(t)=\mathrm{e}^{t \Delta} u_{0}+\mathcal{B}(u, u)(t) .
$$

Above, $\mathrm{e}^{t \Delta}$ denotes the heat semigroup on $\mathbb{R}^{d}$, and the bilinear form $\mathcal{B}$ is defined by

$$
\mathcal{B}(u, w)(t)=\int_{0}^{t} \nabla \mathrm{e}^{(t-s) \Delta}\left(u \nabla(-\Delta)^{-1} w\right)(s) \mathrm{d} s .
$$

Here, we consider arbitrary sign changing and not necessarily radially symmetric solutions. A new result in this spirit is below, with its proof similar to reasonings in [5, Proposition 1, Theorem 1] based on the Morrey space norms counterparts of the $L^{p}-L^{q}$ estimates for the heat semigroup in, e.g., 65 and [39. Note that, in general, we have only weak convergence of $\mathrm{e}^{t \Delta} u_{0}$ to the initial data $u_{0} \in M^{p}\left(\mathbb{R}^{d}\right)$ while $u(t)$ is norm continuous for $t \in(0, T)$. Thus, we are obliged to consider weakly continuous $\left(\mathcal{C}_{\mathrm{w}}\right)$ instead of more natural norm continuous $(\mathcal{C})$ functions.

Proposition 3.1 ([23]).

(i) Given $u_{0} \in M^{d / 2}\left(\mathbb{R}^{d}\right) \cap M^{p}\left(\mathbb{R}^{d}\right)$ with $d \geq 2$ and $p \in\left(\frac{d}{2}, d\right)$, there exist $T=T\left(u_{0}\right)>0$ and a unique local-in-time solution

$$
u \in \mathcal{C}_{\mathrm{w}}\left([0, T], M^{d / 2}\left(\mathbb{R}^{d}\right) \cap M^{p}\left(\mathbb{R}^{d}\right)\right)
$$

of problem (1.1)-1.3 in the sense of (3.1).

(ii) Moreover, if $u_{0} \in M^{d / 2}\left(\mathbb{R}^{d}\right)$ is sufficiently small then $T$ can be chosen arbitrarily large so that the solution is global-in-time:

$$
u \in \mathcal{C}_{\mathrm{w}}\left([0, \infty), M^{d / 2}\left(\mathbb{R}^{d}\right)\right)
$$

and enjoys the decay and regularity property $\sup _{t>0} t^{\beta}|u(t)|_{M^{r}}<\infty$ for $r>d$ and $\beta=\frac{d}{2}\left(\frac{1}{p}-\frac{1}{r}\right)$.

The second assumption $u_{0} \in M^{p}$, with some $p>d / 2$, is a kind of regularity assumption that rules out local singularities stronger than or equal to $\frac{1}{|x|^{2}}$. Indeed, $\mathbb{I}_{\{|x|<R\}} u_{C} \notin M^{p}\left(\mathbb{R}^{d}\right)$ while $\mathbb{I}_{\{|x|>R\}} u_{C} \in M^{p}\left(\mathbb{R}^{d}\right)$. 
Similarly as in previous works, the existence of global-in-time solutions with small data is shown in critical spaces, i.e., those which are scale-invariant under the natural scaling (see, e.g., [5, 13, 48, 53])

$$
u_{\lambda}(x, t)=\lambda^{2} u\left(\lambda x, \lambda^{2} t\right) \text { for each } \lambda>0 .
$$

A much more technical reasoning, that involves Morrey spaces modelled on weak-Lebesgue (i.e. Marcinkiewicz) spaces (see [53]), shows that small initial conditions in $M^{d / 2}\left(\mathbb{R}^{d}\right)$ also lead to global in time solutions. Caution: even local solutions cannot evolve from (very) big data with singularities in the space $M^{d / 2}\left(\mathbb{R}^{d}\right)$, and for data of intermediate size in $M^{d / 2}\left(\mathbb{R}^{d}\right)$ the Cauchy problem is ill-posed. For instance, there is no property of continuity with respect to the initial data, see [26, 29].

We skip the discussion of an interesting structure of steady states and selfsimilar solutions referring the readers to [7, 5, 8] and to a synthesis in [23, Appendices].

\section{Blowup in higher dimensions}

First proofs of blowup of solutions appeared in [47, 27, 18, 6, 77, 56, 57. If $d \geq 3$, a sufficient condition for blowup for a (not necessarily radial) initial condition is that $u_{0}$ is highly concentrated, namely

$$
\left(\frac{\int_{\mathbb{R}^{d}}|x|^{\gamma} u_{0}(x) \mathrm{d} x}{\int_{\mathbb{R}^{d}} u_{0}(x) \mathrm{d} x}\right)^{\frac{d-2}{\gamma}} \leq \tilde{c}_{d, \gamma} M
$$

for some $0<\gamma \leq 2$ and a (small, explicit) constant $\tilde{c}_{d, \gamma}>0$, see [19, (2.4)]. Since

$$
\left|u_{0}\right|_{M^{d / 2}} \geq \tilde{C}_{d, \gamma} M\left(\frac{M}{\int_{\mathbb{R}^{d}}|x|^{\gamma} u_{0}(x) \mathrm{d} x}\right)^{\frac{d-2}{\gamma}}
$$

for some constant $\tilde{C}_{d, \gamma}>0$ and all $u_{0} \in M^{d / 2} \cap L^{1}$, see [19, (2.6)], this means that the Morrey space $M^{d / 2}$ norm of $u_{0}$ satisfying 3.2 is (very!) large:

$$
\left|u_{0}\right|_{M^{d / 2}} \geq \frac{\tilde{C}_{d, 2}}{\tilde{c}_{d, 2}} .
$$

According to [6], $\tilde{c}_{d, 2}=\left(2^{d / 2} d \sigma_{d}\right)^{-1}$ and $\frac{\tilde{C}_{d, 2}}{\tilde{c}_{d, 2}}=\left(\frac{d-2}{d}\right)^{d / 2-1} 2^{d / 2} \sigma_{d} \approx \frac{2^{d / 2}}{\mathrm{e}} \sigma_{d}$.

Recently, some new results on the blowup of solutions to problem (1.1)(1.3) appeared in [24, 25, 14, 26] with some new proofs involving local momenta 
(as in [52]) of (most frequently) radial solutions, and with improved sufficient conditions in terms of the initial datum $u_{0}$. A different kind of blowup (of the Fourier transform of solution) was observed in [12].

\section{Continuation of radially symmetric solutions with moderate size in the critical Morrey space $M^{d / 2}\left(\mathbb{R}^{d}\right)$ norm}

The main result in this direction is

THEOREM 4.1 ([26]). If a radially symmetric initial condition $u_{0} \in$ $M^{d / 2}\left(\mathbb{R}^{d}\right) \cap M^{p}\left(\mathbb{R}^{d}\right)$ with some $p \in\left(\frac{d}{2}, d\right)$ satisfies $\left\|u_{0}\right\|<2 \sigma_{d}$, that is

$$
\sup _{R>0} R^{2-d} \int_{\{|x|<R\}} u_{0}(x) \mathrm{d} x<2 \sigma_{d} \equiv R^{2-d} \int_{\{|x|<R\}} u_{C}(x) \mathrm{d} x,
$$

then each solution $u$ of problem $1.1--1.3$ such that $R^{2-d} \int_{\{|x|<R\}} u(x, t) \mathrm{d} x \in$ $\mathcal{C}^{2}\left((0, T) \times \mathbb{R}^{2}\right)$, can be continued to a global-in-time one, satisfying the bound

$$
\|u(t)\|=\sup _{R>0} R^{2-d} \int_{\{|x|<R\}} u(x, t) \mathrm{d} x<2 \sigma_{d} \text { for every } R>0, t>0 .
$$

Moreover,

$$
\|u(t)\|=\lim _{t \rightarrow \infty} R^{2-d} \int_{\{|x|<R\}} u(x, t) \mathrm{d} x \rightarrow 0 \quad \text { as } \quad t \rightarrow \infty .
$$

For $d \geq 6$ and $\left\|u_{0}\right\|<2 \sigma_{d}$, the $L^{2}$ estimate: $\frac{\mathrm{d}}{\mathrm{d} t}\|u\|_{2}^{2}+\mu\|\nabla u\|_{2}^{2} \leq 0$ holds. If, additionally, $\|u(t)\| \leq \epsilon 2 \sigma_{d}$ for some $0<\epsilon<\frac{d-2}{d}$, then also $\lim _{t \rightarrow \infty}\|u(t)\|_{p}=0$ for $p$ satisfying $\frac{d}{2}<p<\frac{d-2}{2 \epsilon}$.

The assumption on $u_{0}$ in Theorem 4.1 reads in terms of the concentration (and thus the Morrey space $M^{d / 2}\left(\mathbb{R}^{d}\right)$ norm): $\left\|u_{0}\right\|<\epsilon 2 \sigma_{d}$ for some $\epsilon \in(0,1)$. Notice that $\left|u_{C}\right|_{M^{d / 2}}=\left\|u_{C}\right\|=2 \sigma_{d}$ for the Chandrasekhar solution (1.4) so that this singular solution has a regular, bounded (even the constant one) auxiliary function

$$
z(r, t)=r^{2-d} M(r, t)
$$


Here, the radial distribution function $M=M(r, t)$ of a radial solution $u=$ $u(x, t)$ is defined in $(2.2)$ so that $M$ satisfies the equation (equivalent to equation (2.3)

$$
\frac{\partial M}{\partial t}=M_{r r}-\frac{d-1}{r} M_{r}+\frac{1}{\sigma_{d}} r^{1-d} M M_{r}
$$

cf. e.g. [18, and for the radial function $u$ the equality $u(x)=\frac{1}{\sigma_{d}} r^{1-d} \frac{\partial}{\partial r} M(r)$ is satisfied for each $|x|=r$.

Note that for $d=2$ this theorem gives a nonoptimal result: the global-intime existence for $M<4 \pi$ rather than for the optimal range $M<8 \pi$.

\section{Solutions blowing up in a finite time}

We will revisit the classical proof of blowup for the nonlinear heat equation in the seminal paper [38] by H. Fujita, and improve the sufficient conditions for the blowup mentioned before in Section 2, cf. [29].

The key observation is that for a radially symmetric function $u \in L_{\mathrm{loc}}^{1}\left(\mathbb{R}^{d}\right)$ and $v=E_{d} * u$ with $E_{2}(x)=-\frac{1}{2 \pi} \log |x|$ and $E_{d}(x)=\frac{1}{(d-2) \sigma_{d}}|x|^{2-d}$ for $d \geq 3$, solving the Poisson equation $\Delta v+u=0$, the identity

$$
\nabla v(x) \cdot x=-\frac{1}{\sigma_{d}}|x|^{2-d} \int_{\{|y| \leq|x|\}} u(y) \mathrm{d} y
$$

holds as a consequence of the Gauss theorem. Indeed, for the distribution function $M$ of $u$, we have

$$
M(R) \equiv \int_{\{|y| \leq R\}} u(y) \mathrm{d} y=-\int_{\{|y|=R\}} \nabla v(y) \cdot \frac{y}{|y|} \mathrm{d} S .
$$

Thus, for the radial function $\nabla v(x) \cdot \frac{x}{|x|}$ and $|x|=R$, we obtain the required identity

$$
\nabla v(x) \cdot x=\frac{1}{\sigma_{d}} R^{2-d} \int_{\{|y|=R\}} \nabla v(y) \cdot \frac{y}{|y|} \mathrm{d} S=-\frac{1}{\sigma_{d}} R^{2-d} M(R) .
$$

Now, we proceed to apply a classical idea of blowup proof of Fujita. 
THEOREM 5.1. Let $d \geq 2$. If the inequality $T \mathrm{e}^{T \Delta} u_{0}(0)>C(d)$ holds with an explicit constant $C(d) \in[1,2)$, see (5.5) below, then every radial (either classical or weak) solution of problem (1.1)-(1.3) blows up not later than $t=T$, i.e., $\lim _{t \nearrow T}\|u(t)\|_{\infty}=\infty$.

Proof. For a fixed $T>0$ consider the weight function $G=G(x, t)$, $x \in \mathbb{R}^{d}, t \in[0, T)$, which solves the backward heat equation with the unit measure as the final time condition

$$
G_{t}+\Delta G=0, \quad G(., T)=\delta_{0} .
$$

Clearly, we have a (unique nonnegative) solution

$$
G(x, t)=(4 \pi(T-t))^{-\frac{d}{2}} \exp \left(-\frac{|x|^{2}}{4(T-t)}\right),
$$

defined by the Gauss-Weierstrass kernel, satisfying $\int G(x, t) \mathrm{d} x=1$, and moreover,

$$
\nabla G(x, t)=-\frac{x}{2(T-t)} G(x, t)
$$

Define for a solution $u$ of $(1.1)-(1.2)$, which is supposed to exist on $[0, T)$, the moment

$$
W(t)=\int G(x, t) u(x, t) \mathrm{d} x .
$$

Since $G$ decays exponentially fast in $x$ as $|x| \rightarrow \infty$, the moment $W$ is well defined even for a wider class of solutions $u=u(x, t)$ polynomially bounded in $x$.

The evolution of the moment $W$ is governed by the differential identity

$$
\begin{aligned}
\frac{\mathrm{d} W}{\mathrm{~d} t} & =\int G u_{t} \mathrm{~d} x+\int G_{t} u \mathrm{~d} x \\
& =\int(\Delta u-\nabla \cdot(u \nabla v)) G \mathrm{~d} x-\int \Delta G u \mathrm{~d} x \\
& =\int \Delta G u \mathrm{~d} x+\int u \nabla v \cdot \nabla G \mathrm{~d} x-\int \Delta G u \mathrm{~d} x \\
& =-\frac{1}{2(T-t)} \int u \nabla v \cdot x G \mathrm{~d} x \\
& =\frac{1}{2 \sigma_{d}(T-t)} \int u(x, t) M(|x|, t)|x|^{2-d} G(x, t) \mathrm{d} x
\end{aligned}
$$




$$
\begin{aligned}
& =\frac{\sigma_{d}}{2 \sigma_{d}(T-t)} \int_{0}^{\infty} \frac{1}{\sigma_{d}} M_{r}(r, t) r^{1-d} M(r, t) r^{2-d} G(r, t) r^{d-1} \mathrm{~d} r \\
& =\frac{1}{2 \sigma_{d}(T-t)} \int_{0}^{\infty} M_{r} M r^{2-d} G \mathrm{~d} r \\
& =-\frac{1}{4 \sigma_{d}(T-t)} \int_{0}^{\infty} M^{2}\left(r^{2-d} G\right)_{r} \mathrm{~d} r \\
& =\frac{1}{4 \sigma_{d}(T-t)} \int_{0}^{\infty} M^{2} r^{1-d}\left((d-2)+\frac{r^{2}}{2(T-t)}\right) G \mathrm{~d} r,
\end{aligned}
$$

where we used the radial symmetry of the solution $u$ in $(5.2)$, identity (5.1) and, of course, the radial symmetry of $G$.

Expressing the moment $W$ in the radial variables we obtain

$$
\begin{aligned}
W(t) & =\sigma_{d} \int_{0}^{\infty} \frac{1}{\sigma_{d}} M_{r} r^{1-d} G r^{d-1} \mathrm{~d} r=-\int_{0}^{\infty} M G_{r} \mathrm{~d} r \\
& =\int_{0}^{\infty} M \frac{r}{2(T-t)} G \mathrm{~d} r .
\end{aligned}
$$

Now, applying the Cauchy inequality to the quantity (5.3), we get

$$
\begin{aligned}
W^{2}(t)= & \left(\int_{0}^{\infty} M \frac{r}{2(T-t)} G \mathrm{~d} r\right)^{2} \\
\leq & \int_{0}^{\infty} M^{2} r^{1-d}\left((d-2)+\frac{r^{2}}{2(T-t)}\right) G \mathrm{~d} r \\
& \times \frac{1}{2(T-t)} \int_{0}^{\infty} \frac{r^{d+1} G}{r^{2}+2(d-2)(T-t)} \mathrm{d} r .
\end{aligned}
$$

Returning to the time derivative of $W$ in equation 5.2 , we arrive at the differential inequality

$$
\begin{aligned}
\frac{\mathrm{d} W}{\mathrm{~d} t} & \geq \frac{1}{4 \sigma_{d}(T-t)} W^{2}(t)\left(\int_{0}^{\infty} \frac{r^{d+1}}{2(T-t)} \frac{G}{r^{2}+2(d-2)(T-t)} \mathrm{d} r\right)^{-1} \\
& =\frac{\pi^{\frac{d}{2}}}{8 \sigma_{d}} W^{2}(t)\left(\int_{0}^{\infty} \varrho^{d+1}\left(2(d-2)+4 \varrho^{2}\right)^{-1} \mathrm{e}^{-\varrho^{2}} \mathrm{~d} \varrho\right)^{-1}
\end{aligned}
$$

where $\varrho=\frac{r}{2(T-t)^{1 / 2}}$. Recalling 2.4 , we denote

$$
C(d)=\frac{16}{\Gamma\left(\frac{d}{2}\right)} \int_{0}^{\infty} \varrho^{d+1}\left(2(d-2)+4 \varrho^{2}\right)^{-1} \mathrm{e}^{-\varrho^{2}} \mathrm{~d} \varrho .
$$


Clearly, $C(2)=2$, and $C(d)<2$ for $d \geq 3$, since we have

$$
C(d)<\frac{16}{\Gamma\left(\frac{d}{2}\right)} \int_{0}^{\infty} \frac{1}{4} \varrho^{d-1} \mathrm{e}^{-\varrho^{2}} \mathrm{~d} \varrho=\frac{4}{\Gamma\left(\frac{d}{2}\right)} \frac{1}{2} \int_{0}^{\infty} \tau^{\frac{d}{2}-1} \mathrm{e}^{-\tau} \mathrm{d} \tau=2
$$

Thus, we finally obtain

$$
\frac{\mathrm{d} W}{\mathrm{~d} t} \geq \frac{1}{C(d)} W^{2}(t)
$$

which, after an integration, leads to

$$
W(t) \geq\left(\frac{1}{W(0)}-\frac{t}{C(d)}\right)^{-1}
$$

Now, it is clear that if initially

$$
W(0)=\mathrm{e}^{T \Delta} u_{0}(0)>\frac{C(d)}{T}
$$

is satisfied, then $\limsup W(t)=\infty$, which means that $\limsup u(x, t)=\infty$ and completes the proof.

Note that the blowup rate is such that $\liminf _{t \nearrow T}(T-t) W(t)>0$. For other results on blowup rates (e.g., a faster blowup, i.e., of the II type), see [40, 55, 54].

Observe that the equality in the Cauchy inequality (5.4) holds if and only if

$$
0 \leq M(r, t)=\frac{A(t) r^{d}}{r^{2}+2(d-2)(T-t)}=(T-t)^{\frac{d}{2}-1} \frac{A(t) 2^{d} \varrho^{d}}{4 \varrho^{2}+2(d-2)}
$$

with some $A(t) \geq 0$. Consequently, inequality (5.6) becomes

$$
W(t)=\left(\frac{1}{W(0)}-\frac{t}{C(d)}\right)^{-1}
$$

and if $d \geq 3$

$$
\begin{aligned}
W(0) & =\frac{1}{2 T} \int_{0}^{\infty} \frac{A(0) r^{d+1}}{r^{2}+2(d-2) T} \mathrm{e}^{-r^{2} /(4 T)}(4 \pi T)^{-\frac{d}{2}} \mathrm{~d} r \\
& =\frac{A(0)}{T} \frac{\Gamma\left(\frac{d}{2}\right)}{8 \pi^{\frac{d}{2}}} C(d) \geq \frac{C(d)}{T}
\end{aligned}
$$


then the solution blows up not later than $T$. This holds exactly when $A(0) \geq$ $4 \sigma_{d}$. This solution (cf. [33, (33)]) satisfies identity 5.8 with $W(0)=\frac{C(d)}{T}$, and is, in a sense, a kind of the minimal smooth blowing up solution, i.e., it gives a lower bound on blowing up solutions. So, we have an explicit example of blowing up solution with infinite mass

$$
M(r, t)=\frac{4 \sigma_{d} r^{d}}{r^{2}+2(d-2)(T-t)}
$$

whose density approaches $\frac{4(d-2)}{|x|^{2}}=2 u_{C}(x)$, i.e., twice the singular stationary solution, when $t \nearrow T$, so that the density of the solution becomes infinite at the origin for $t=T$. The corresponding initial density is, of course,

$$
\begin{gathered}
u_{0}(x)=4(d-2) \frac{r^{2}+2 T}{\left(r^{2}+2(d-2) T\right)^{2}}, \\
\left|u_{0}\right|_{M^{d / 2}}=4 \sigma_{d}=\lim _{r \rightarrow \infty} r^{2-d} M(r, t)=|u(t)|_{M^{d / 2}} .
\end{gathered}
$$

We give below some other examples of initial data leading to a finite time blowup of solutions.

REMARK 5.2. Observe that for each initial condition $u_{0} \not \equiv 0$ there is $N>0$ such that condition (5.7) is satisfied for $N u_{0}$.

Clearly, by $\left\|u_{C}\right\|=\left|u_{C}\right|_{M^{d / 2}}=2 \sigma_{d}$, for each $\eta>2$ the solution with the initial condition $u_{0}=\eta u_{C}$ blows up. Moreover, for each $\eta>2$ and sufficiently large $R=R(\eta)>1$ the bounded initial condition of compact support $u_{0}=$ $\eta \mathbb{1}_{\{1 \leq|x| \leq R\}} u_{C}$ leads to a blowing up solution, see (5.7). The singularity of that solution at the blowing up time is $\sim \frac{1}{|x|^{2}}$ at the origin. It seems that the latter result cannot be obtained applying previously known sufficient criteria for blowup like (3.2).

On the other hand, the initial data like $\min \left\{1, u_{C}\right\}+\epsilon \psi$ with a smooth nonnegative, compactly supported function $\psi$ and a sufficiently small $\epsilon>0$ (they are somewhere above the critical $u_{C}$ pointwisely) lead to global-in-time solutions according to Theorem 4.1 .

Sufficient conditions for blowup for radial $u_{0} \geq 0$ mentioned above

$-\sup _{t>0} t \mathrm{e}^{t \Delta} u_{0}(0) \gg 1$,

$-\sup _{t>0} t\left\|\mathrm{e}^{t \Delta} u_{0}\right\|_{\infty} \gg 1$,

- $\left\|u_{0}\right\| \equiv \sup _{r>0} r^{2-d} \int_{\{|x|<r\}} u_{0}(x) \mathrm{d} x \gg 1$,

$-\|\left. u_{0}\right|_{M^{d / 2}} \equiv \sup _{r>0, x \in \mathbb{R}^{d}} r^{2-d} \int_{\{|y-x|<r\}} u_{0}(y) \mathrm{d} y \gg 1$, 
are mutually equivalent. Note that, however, some of these equivalences are rather nontrivial and the comparison constants for pairs of those quantities strongly depend on $d$.

REMARK 5.3 (Initial traces). One can show, along the lines of the reasonings in [1, (1.4), Proposition 4.3], that for $d \geq 3$ each nonnegative radial solution $u$ on $\mathbb{R}^{d} \times(0, T)$ of system $1.1-1.2$ has the initial trace, that is, $u_{0}=\lim _{t \searrow_{0}} u(t)$ exists in the sense of weak convergence of measures. Moreover, $u_{0}$ is uniformly in $L_{\text {loc }}^{1}\left(\mathbb{R}^{d}\right)$, and the solution $u(t)$ satisfies the uniform bound in the local Morrey space $M_{\mathrm{loc}}^{d / 2}$, i.e., $\|\left. u(t)\right|_{M_{\mathrm{loc}}^{d / 2}} \equiv$ $\sup _{0<r<1, x \in \mathbb{R}^{d}} r^{2-d} \int_{\{|y-x|<r\}}|u(y, t)| \leq J(d)<\infty$ for all $t \in(0, T)$. This, supplemented with Corollary 5.4 below, shows that the spaces $M^{d / 2}\left(\mathbb{R}^{d}\right)$ are nearly the optimal ones with respect to the local- and global-in-time solvability of system $1.1-1.2$.

Thus, our results for radially symmetric solutions (which we suppose to exist) can be summarized in the following dichotomy result.

\section{Corollary 5.4.}

(i) If $u_{0}$ is such that $\left\|u_{0}\right\|<2 \sigma_{d}$ then the solution of problem 1.1 - 1.3 is global-in-time $([23])$;

(ii) if $u_{0}$ is such that $T \mathrm{e}^{T \Delta} u_{0}(0)>2$ (which is the case if either $u_{0}(x)>$ $2 u_{C}(x)$ pointwise for each $x \in \mathbb{R}^{d} \backslash\{0\}$, or by [29, Proposition 4.6], guaranteed by, e.g., $\left\|u_{0}\right\|>2 \sqrt{\pi d} 2 \sigma_{d}$, asymptotically as $d \rightarrow \infty$, so for one $\left.R_{0}>0: R_{0}^{2-d} \int_{\left\{|x|<R_{0}\right\}} u_{0}(x) \mathrm{d} x>2 \sqrt{\pi d} 2 \sigma_{d}\right)$, then the solution of problem 1.1 -1.3 blows up not later than at $t=T$.

\section{Hypercontractivity properties}

A (proto)typical result in this direction is the following.

THEOREM 6.1. Assume that $d \geq 15$. Suppose that $u(x, t)$ is a global-intime radial solution satisfying $0 \leq u(x, t) \leq u_{C}(x)$ for all $x \in \mathbb{R}^{d}$ and $t \geq 0$. There exists $1<p_{0}<2$ such that if $u_{C}-u_{0} \in L^{2}\left(\mathbb{R}^{d}\right) \cap L^{p}\left(\mathbb{R}^{d}\right)$ with some $p \in\left(p_{0}, 2\right)$, then

$$
\left\|u_{C}-u(t)\right\|_{2} \leq\left\|u_{C}-u_{0}\right\|_{2}
$$


and

$$
\left\|u_{C}-u(t)\right\|_{2} \leq C(p, d) t^{-\frac{d}{2}\left(\frac{1}{p}-\frac{1}{2}\right)}\left\|u_{C}-u_{0}\right\|_{p}
$$

for all $t>0$ and a number $C(p, d)$ independent of $t$ and $u$.

The question of the existence of solutions with $0 \leq u(x, t) \leq u_{C}(x)$ is nontrivial, and the proof involves an approximation procedure by solutions like those in Proposition 3.1 and Theorem 4.1.

To prove Theorem 6.1 we need a few ingredients including the hypercontractivity of the semigroup of linear operators $\mathrm{e}^{-t \mathcal{L}}$ describing the evolution in vicinity of the singular solution $u_{C}$, and a perturbation result, cf. an analogous scheme in [60, 59] in the case of nonlinear heat equations.

Here, we sketch the first part of those constructions. We consider the linearization of problem (1.1) - 1.3), and we substitute $w(x, t)=u_{C}(x)-$ $u(x, t), \Delta \varphi_{C}+u_{C}=0$ to 1.1 to get

$$
\begin{aligned}
w_{t} & =\Delta w-\nabla \cdot\left(u_{C} \nabla \varphi\right)-\nabla \cdot\left(w \nabla \varphi_{C}\right)+\nabla \cdot(w \nabla \varphi), \\
\Delta \varphi+w & =0 \\
w(x, 0) & =w_{0}(x) .
\end{aligned}
$$

Let us define the linear differential operator

$$
\mathcal{L} w=-\Delta w+\nabla u_{C} \cdot \nabla \varphi-u_{C} w+\nabla \cdot\left(w \nabla \varphi_{C}\right) .
$$

In the following, we study properties of the operator $\mathcal{L}$, see an analogous approach in [59, 60] for nonlinear heat equations.

LEMMA 6.2.

(i) Assume that $d \geq 15$. Then there exists a constant $\lambda>0$ such that the operator $\mathcal{L}$ defined in 6.2 satisfies the following inequality

$$
\langle\mathcal{L} w, w\rangle \geq \lambda\|\nabla w\|_{2}^{2}
$$

for all radial functions $w \in H^{1}\left(\mathbb{R}^{d}\right)$.

(ii) Assume that $d \geq 17$. Then inequality 6.3 holds for another $\lambda>0$ and each $w \in H^{1}\left(\mathbb{R}^{\bar{d}}\right)$. 
(i) This is a consequence of the identity

$$
\begin{aligned}
\langle\mathcal{L} w, w\rangle & =\|\nabla w\|_{2}^{2}+\int_{\mathbb{R}^{d}} \nabla u_{C} \cdot \nabla \varphi w \mathrm{~d} x-\frac{3}{2} \int_{\mathbb{R}^{d}} u_{C} w^{2} \mathrm{~d} x \\
& =\|\nabla w\|_{2}^{2}-4(d-2) \int_{\mathbb{R}^{d}} \frac{x}{|x|^{4}} \cdot \nabla \varphi w \mathrm{~d} x-3(d-2) \int_{\mathbb{R}^{d}} \frac{w^{2}}{|x|^{2}} \mathrm{~d} x
\end{aligned}
$$

obtained by multiplying equation $w_{t}+\mathcal{L} w=0$ by $w$ and integrating by parts.

Then, the Hardy inequality (cf. [2])

$$
\frac{(d-2)^{2}}{4}\left\|\frac{f}{|x|}\right\|_{2}^{2} \leq\|\nabla f\|_{2}^{2},
$$

and positivity of the middle term for radial functions $w$ of arbitrary sign (which is a rather subtle property) prove inequality 6.3 if $1-\frac{12}{d-2}>0$, i.e., $d \geq 15$, holds.

(ii) An alternative approach to the property of hypercontractivity of the linearization operator $\mathcal{L}$ is obtained by estimating the middle term in formula (6.4)

$$
\begin{aligned}
\left|4(d-2) \int \frac{x}{|x|^{4}} \cdot \nabla \varphi w \mathrm{~d} x\right| & \leq 4(d-2) \int \frac{|\nabla \varphi|}{|x|^{2}} \frac{|w|}{|x|} \mathrm{d} x \\
& \leq 4(d-2) \frac{4}{d(d-4)} \frac{2}{d-2}\|\nabla w\|_{2}^{2}
\end{aligned}
$$

by the Hardy inequality, and then by the Rellich inequality

$$
\frac{d^{2}(d-4)^{2}}{16}\left\|\frac{f}{|x|^{2}}\right\|_{2}^{2} \leq\|\Delta f\|_{2}^{2},
$$

see $[2,(6),(6.2 .3)]$. To conclude, observe that $\frac{32}{d(d-4)}<1-\frac{12}{d-2}$ is valid for $d \geq 17$.

Then, $\mathrm{e}^{-t \mathcal{L}}$ is shown to be a holomorphic semigroup on $L^{2}\left(\mathbb{R}^{d}\right)$. The other ingredients of the proof are technical and include global existence for quadratic perturbation (6.1), decay estimates based on the properties of the semigroup $\mathrm{e}^{-t \mathcal{L}}$, etc.

Acknowledgements. It is my honor and pleasure to thank my teacher and advisor Andrzej Krzywicki and my friend Tadeusz Nadzieja for introducing me in the topic of mean field models in statistical mechanics, and all my friends for fruitful collaboration over years: Waldemar Hebisch, Jacek Dziubański, Danielle Hilhorst, Andrzej Raczyński, Wojbor Woyczyński, Philippe 
Laurençot, Jean Dolbeault, Maria J. Esteban, Marco Cannone, Ignacio Guerra, Robert Stańczy, Peter A. Markowich, Lorenzo Brandolese, Lucilla Corrias, Gang Wu, Eduardo Elio Espejo, Noriko Mizoguchi, Jacek Zienkiewicz, Tomasz Cieślak, Dominika Pilarczyk, Xiaoxin Zheng (in a chronological order of starting joint research), with the special affection for Grzegorz Karch.

\section{References}

[1] Andreucci D., DiBenedetto E., On the Cauchy problem and initial traces for a class of evolution equations with strongly nonlinear sources, Ann. Scuola Norm. Sup. Pisa Cl. Sci. (4) 18 (1991), 363-441.

[2] Balinsky A.A., Evans W.D., Lewis R.T., The Analysis and Geometry of Hardy's Inequality, Universitext, Springer, Cham, 2015.

[3] Bedrossian J., Masmoudi N., Existence, uniqueness and Lipschitz dependence for Patlak-Keller-Segel and Navier-Stokes in $\mathbb{R}^{2}$ with measure-valued initial data, Arch. Ration. Mech. Anal. 214 (2014), 717-801.

[4] Bellomo N., Bellouquid A., Tao Y., Winkler M., Toward a mathematical theory of Keller-Segel models of pattern formation in biological tissues, Math. Models Methods Appl. Sci. 25 (2015), 1663-1763.

[5] Biler P., The Cauchy problem and self-similar solutions for a nonlinear parabolic equation, Studia Math. 114 (1995), 181-205.

[6] Biler P., Existence and nonexistence of solutions for a model of gravitational interaction of particles. III, Colloq. Math. 68 (1995), 229-239.

[7] Biler P., Growth and accretion of mass in an astrophysical model, Appl. Math. (Warsaw) 23 (1995), 179-189.

[8] Biler P., Local and global solvability of parabolic systems modelling chemotaxis, Adv. Math. Sci. Appl. 8 (1998), 715-743.

[9] Biler P., Radially symmetric solutions of a chemotaxis model in the plane - the supercritical case, in: Rencławowicz J., Zajączkowski W.M. (eds.), Parabolic and NavierStokes Equations. Part 1, Banach Center Publications, 81, Polish Acad. Sci. Inst. Math., Warsaw, 2008, pp. 31-42.

[10] Biler P., Blowup versus global in time existence of solutions for nonlinear heat equations, Topol. Methods Nonlinear Anal. To appear. Available at arXiv:1705.03931v2

[11] Biler P., Solvability for nonlinear heat equations with fractional diffusion. In preparation.

[12] Biler P., Brandolese L., Global existence versus blow up for some models of interacting particles, Colloq. Math. 106 (2006), 293-303.

[13] Biler P., Brandolese L., On the parabolic-elliptic limit of the doubly parabolic KellerSegel system modelling chemotaxis, Studia Math. 193 (2009), 241-261.

[14] Biler P., Cieślak T., Karch G., Zienkiewicz J., Local criteria for blowup in twodimensional chemotaxis models, Discrete Contin. Dyn. Syst. 37 (2017), 1841-1856.

[15] Biler P., Corrias L., Dolbeault J., Large mass self-similar solutions of the parabolicparabolic Keller-Segel model of chemotaxis, J. Math. Biol. 63 (2011), 1-32.

[16] Biler P., Dolbeault J., Long time behavior of solutions of Nernst-Planck and DebyeHückel drift-diffusion systems, Ann. Henri Poincaré 1 (2000), 461-472.

[17] Biler P., Guerra I., Karch G., Large global-in-time solutions of the parabolic-parabolic Keller-Segel system on the plane, Commun. Pure Appl. Anal. 14 (2015), 2117-2126.

[18] Biler P., Hilhorst D., Nadzieja T., Existence and nonexistence of solutions for a model of gravitational interaction of particles. II, Colloq. Math. 67 (1994), 297-308. 
[19] Biler P., Karch G., Blowup of solutions to generalized Keller-Segel model, J. Evol. Equ. 10 (2010), 247-262.

[20] Biler P., Karch G., Solutions of fractional chemotaxis models. In preparation.

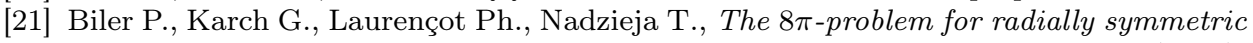
solutions of a chemotaxis model in a disc, Topol. Methods Nonlinear Anal. 27 (2006), 133-147.

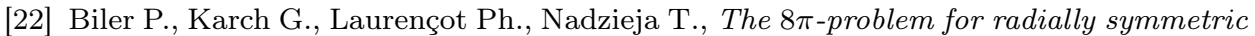
solutions of a chemotaxis model in the plane, Math. Methods Appl. Sci. 29 (2006), 1563-1583.

[23] Biler P., Karch G., Pilarczyk D., Global radial solutions in classical Keller-Segel chemotaxis model. In preparation.

[24] Biler P., Karch G., Zienkiewicz J., Optimal criteria for blowup of radial and $N$-symmetric solutions of chemotaxis systems, Nonlinearity $\mathbf{2 8}$ (2015), 4369-4387.

[25] Biler P., Karch G., Zienkiewicz J., Morrey spaces norms and criteria for blowup in chemotaxis models, Netw. Heterog. Media $1 \overline{1}(2016), 239-\overline{250 .}$

[26] Biler P., Karch G., Zienkiewicz J., Large global-in-time solutions to a nonlocal model of chemotaxis, Adv. Math. 330 (2018), 834-875.

[27] Biler P., Nadzieja T., Existence and nonexistence of solutions for a model of gravitational interaction of particles. I, Colloq. Math. 66 (1994), 319-334.

[28] Biler P., Zienkiewicz J., Existence of solutions for the Keller-Segel model of chemotaxis with measures as initial data, Bull. Pol. Acad. Sci. Math. 63 (2015), $41-\overline{5} 1$.

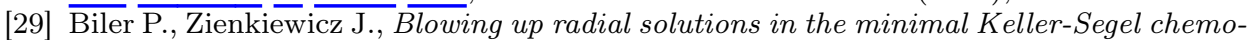
taxis model. In preparation.

[30] Blanchet A., Carlen E.A., Carrillo J.A., Functional inequalities, thick tails and asymptotics for the critical mass Patlak-Keller-Segel model, J. Funct. Anal. 262 (2012), $2142-2230$.

[31] Blanchet A., Carrillo J.A., Masmoudi N., Infinite time aggregation for the critical Patlak-Keller-Segel model in $\mathbb{R}^{2}$, Comm. Pure Appl. Math. 61 (2008), 1449-1481.

[32] Blanchet A., Dolbeault J., Perthame B., Two-dimensional Keller-Segel model: optimal critical mass and qualitative properties of the solutions, Electron. J. Differential Equations 2006, no. 44, 32 pp.

[33] Brenner M.P., Constantin P., Kadanoff L.P., Schenkel A., Venkataramani S.C., Diffusion, attraction and collapse, Nonlinearity 12 (1999), 1071-1098.

[34] Chandrasekhar S., Principles of Stellar Dynamics, University of Chicago Press, Chicago, 1942.

[35] Chavanis P.H., Sommeria J., Robert R., Statistical mechanics of two-dimensional vortices and collisionless stellar systems, The Astrophys. Journal 471 (1996), 385-399.

[36] Corrias L., Perthame B., Zaag H., Global solutions of some chemotaxis and angiogenesis systems in high space dimensions, Milan J. Math. 72 (2004), 1-28.

[37] Debye P., Hückel E., Zur Theorie der Electrolyte. II, Phys. Zft. 24 (1923), 305-325.

[38] Fujita H., On the blowing up of solutions of the Cauchy problem for $u_{t}=\Delta u+u^{1+\alpha}$, J. Fac. Sci. Univ. Tokyo Sect. I 13 (1966), 109-124.

[39] Giga Y., Miyakawa T., Navier-Stokes flow in $\mathbb{R}^{3}$ with measures as initial vorticity and Morrey spaces, Comm. Partial Differential Equations 14 (1989), 577-618.

[40] Giga Y., Mizoguchi N., Senba T., Asymptotic behavior of type I blowup solutions to a parabolic-elliptic system of drift-diffusion type, Arch. Ration. Mech. Anal. 201 (2011), 549-573.

[41] Herrero M.A., Velázquez J.J.L., Singularity patterns $\underline{\text { in }}$ a chemotaxis model, Math. Ann. 306 (1996), 583-623.

[42] Herrero M.A., Velázquez J.J.L., Chemotactic collapse for the Keller-Segel model, J. Math. Biol. 35 (1996), 177-194.

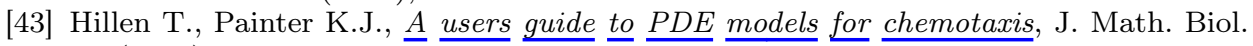
58 (2009), 183-217.

[44] Horstmann D., From 1970 until present: the Keller-Segel model in chemotaxis and its consequences. I, Jahresber. Deutsch. Math.-Verein. 105 (2003), 103-165. 
[45] Horstmann D., From 1970 until present: the Keller-Segel model in chemotaxis and its consequences. II, Jahresber. Deutsch. Math.-Verein. 106 (2004), 51-69.

[46] Iwabuchi T., Global well-posedness for Keller-Segel system in Besov type spaces, J. Math. Anal. Appl. 379 (2011), 930-948.

[47] Jäger W., Luckhaus S., On explosions of solutions to a system of partial differential equations modelling chemotaxis, Trans. Amer. Math. Soc. 329 (1992), 819-824.

[48] Karch G., Scaling in nonlinear parabolic equations, J. Math. Anal. Appl. 234 (1999), $534-558$.

[49] Kavallaris N.I., Souplet Ph., Grow-up rate and refined asymptotics for a twodimensional Patlak-Keller-Segel model in a disk, SIAM J. Math. Anal. 40 (2008/09), $1852-1881$.

[50] Keller E.F., Segel L.A., Initiation of slime mold aggregation viewed as an instability, J. Theor. Biol. 26 (1970), 399-415.

[51] Kozono H., Sugiyama Y., The Keller-Segel system of parabolic-parabolic type with initial data in weak $L^{n / 2}\left(\mathbb{R}^{n}\right)$ and its application to self-similar solutions, Indiana Univ. Math. J. 57 (2008), 1467-1500.

[52] Kurokiba M., Ogawa T., Finite time blow-up of the solution for a nonlinear parabolic equation of drift-diffusion type, Differential Integral Equations 16 (2003), 427-452.

[53] Lemarié-Rieusset P.-G., Small data in an optimal Banach space for the parabolicparabolic and parabolic-elliptic Keller-Segel equations in the whole space, Adv. Differential Equations 18 (2013), 1189-1208.

[54] Mizoguchi N., Senba T., Type-II blowup of solutions to an elliptic-parabolic system, Adv. Math. Sci. Appl. 17 (2007), 505-545.

[55] Mizoguchi N., Senba T., A sufficient condition for type I blowup in a parabolic-elliptic system, J. Differential Equations 250 (2011), 182-203.

[56] Nagai T., Blowup of nonradial solutions to parabolic-elliptic systems modeling chemotaxis in two-dimensional domains, J. Inequal. Appl. 6 (2001), 37-55.

[57] Naito Y., Senba T., Blow-up behavior of solutions to a parabolic-elliptic system on higher dimensional domains, Discrete Contin. Dyn. Syst. 32 (2012), 3691-3713.

[58] Naito Y., Senba T., Bounded and unbounded oscillating solutions to a parabolic-elliptic system in two dimensional space, Commun. Pure Appl. Anal. 12 (2013), 1861-1880.

[59] Pilarczyk D., Asymptotic stability of singular solution to nonlinear heat equation, Discrete Contin. Dyn. Syst. 25 (2009), 991-1001.

[60] Pilarczyk D., Self-similar asymptotics of solutions to heat equation with inverse square potential, J. Evol. Equ. 13 (2013), 69-87.

[61] Quittner P., Souplet Ph., Superlinear Parabolic Problems. Blow-up, Global Existence and Steady States, Birkhäuser Advanced Texts, Birkhäuser, Basel, 2007.

[62] Senba T., Blowup behavior of radial solutions to Jäger-Luckhaus system in high dimensional domains, Funkcial. Ekvac. 48 (2005), 247-271.

[63] Souplet Ph., Morrey spaces and classification of global solutions for a supercritical semilinear heat equation in $\mathbb{R}^{n}$, J. Funct. Anal. 272 (2017), 2005-2037.

[64] Suzuki T., Free Energy and Self-Interacting Particles, Progress in Nonlinear Differential Equations and their Applications, 62, Birkhäuser, Boston, 2005.

[65] Taylor M.E., Analysis on Morrey spaces and applications to Navier-Stokes and other evolution equations, Comm. Partial Differential Equations 17 (1992), 1407-1456.

Institute of Mathematics

UNIVERSITY OF WROCEAW

Pl. GRunWaldzki $2 / 4$

50-384 WroceaW

POLAND

e-mail: Piotr.Biler@math.uni.wroc.pl 